\begin{tabular}{|c|c|c|c|c|c|c|}
\hline & \multicolumn{2}{|c|}{ SF-12 } & \multicolumn{4}{|c|}{ CAPE } \\
\hline & Physical & Mental & Positive & Negative & Depressive & Total \\
\hline SpA & $31.8(13.6)^{\star \star}$ & $43.8(10.6)$ & $1.32(0.2)$ & $1.81(0.4)^{\star}$ & $1.98(0.5)^{\star \star}$ & $1.59(0.3)^{x}$ \\
\hline RA & $33.1(13.1)^{\star \star}$ & $44.9(12.7)$ & $1.37(0.3)$ & $1.66(0.4)$ & $1.86(0.5)^{\star}$ & $1.55(0.3)$ \\
\hline Control & $47.0(16.1)$ & $47.2(7.5)$ & $1.30(0.2)$ & $1.61(0.4)$ & $1.65(0.3)$ & $1.47(0.2)$ \\
\hline
\end{tabular}

differences, according a t de Student test with control group is shown in both (* $\mathrm{p}<0.05,{ }^{* *} \mathrm{p}<0.01$ ).

There were no significant differences in the mental component of the SF-12. These differences appear at the physical component, since patients have impaired their mobility and function due to their disease. About the CAPE questionnaire, patients had a little bit higher score due mainly to the appearance of depressive symptoms. The values of positive symptoms of psychosis remained within the normal range for diseases analyzed.

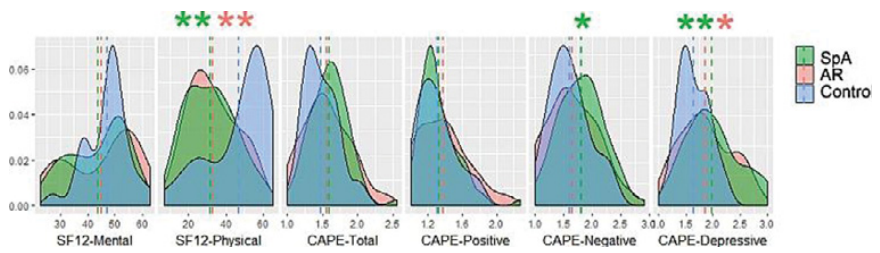

Conclusions: In our study, we found significant differences in the dimensions, especially depressive, of the CAPE scale among patients with rheumatic diseases (especially in $\mathrm{SpA}$ ) and healthy subjects. This gives us an idea of the importance of considering the psychological problems of patients (anxiety, depression, ...) to improve the treatment of rheumatic disease.

Acknowledgements: We would like to thank these patients' organizations for their collaboration in our study: Coordinadora Española de Asociaciones De Espondiloartritis (CEADE), Asociación Cordobesa de Enfermos de Espondilitis (ACEADE), Asociación Cordobesa de Enfermos de Artritis Reumatoide (ACOARE).

Disclosure of Interest: None declared

DOI: 10.1136/annrheumdis-2017-eular.3505

\section{THU0625 DESIGN OF AN INFORMATION AND COMMUNICATIONS TECHNOLOGY PLATFORM TO SUPPORT COORDINATION OF CARE FOR RHEUMATOID ARTHRITIS PATIENTS WITH CARDIOVASCULAR CO-MORBIDITIES - FIRST EXPERIENCES}

J.G. Richter, G. Chehab, M. Schneider on behalf of the PICASO Consortium (www.picaso-project.eu). Policlinic \& Hiller Research Unit for Rheumatology, Heinrich-Heine-University Duesseldorf, Duesseldorf, Germany

Background: Coordination of care plans between healthcare sectors and efficient management of patients (pts) with co-morbidities is of large demand. Rheumatoid arthritis (RA) pts are at increased risk of cardiovascular diseases (CVD). Different stakeholders are potentially involved in the EULAR recommended management processes. Optimized orchestration of accumulated information is of major importance to ensure data quality, meaningful management processes and cost effectiveness. A newly developed information and communications technology (ICT) platform within the Horizon2020-funded PICASO-project (www.picasoproject.eu) will support a continuum of care from hospitals and outpatient clinics to the home.

Objectives: Explore challenges to provide an efficient ICT integrated solution across many healthcare professionals working for various organisations and potentially crossing national borders that complies to privacy and regulatory constraints allowing more efficient care management. Suitable system architecture and appropriate features require identification of target users' user requirements. PICASO platform will be developed and trialed with pts and clinician. The proposed system architecture will be evaluated for suitability for a larger scale rollout.

Methods: Projects' pre-defined clinical and technological driven work packages started. Various stakeholders (e.g. pts, local data security and IT representatives, health care insurances' representatives, clinicians) were integrated in the design phase. A PICASO ethical board including external members (e.g. Chair of the Standing Committee of PARE) addressed ethical and legal concerns.

Results: Current work-flows for the care plan management including stakeholders' hand-over procedures were elaborated. Vision scenarios $(n=11)$ and To-Be Use Cases $(n=18)$ addressing solutions including home monitoring were developed (1). A comprehensive list of user requirements (currently $n=87$ ) resulted. Detailed system architecture descriptions are stipulated. Ethical issues and how to handle these, in particular data-protection and -privacy challenges were pre-assigned as these affect platforms' architecture. PICASO ethical principles and guidelines were stated (2). The platform is under development. The first trial running over nine months including RA-pts will start in spring 2017. First experiences will be reported at EULAR.

Conclusions: Considering the needs of a highly valued, specialised health care system relevant To-Be Use Cases, numerous user requirements and EU-wide ethical and legal issues were gathered to serve as basis for appropriate design, development and implementation of the ICT platform. Software development will take place in iterative cycles followed by prototypes' thoroughly evaluated by real end users investigating usability and acceptance. The platform will become available for RA-pts in routine care but also for wider applicability in Rheumatology and other chronic diseases.

References:

[1] PICASO Consortium (2016), D2.1 Scenarios and Use Cases for Integrated Care. http://www.picaso-project.eu/download/4894

[2] PICASO Consortium (2016), D3.3 PICASO Ethical Guidelines. http://www. picaso-project.eu/download/520/; both last accessed 27/01/2017.

Acknowledgements: This project has received funding from the European Union's Horizon 2020 research and innovation programme under grant agreement No 689209.

Disclosure of Interest: None declared

DOI: 10.1136/annrheumdis-2017-eular.4001

\section{THU0626 COST-EFFECTIVENESS OF EARLY TREATMENT OF ACPA POSITIVE RHEUMATOID ARTHRITIS PATIENTS WITH ABATACEPT}

A.S. Neubauer ${ }^{1}$, C. Minartz ${ }^{1}$, K.H. Herrmann ${ }^{2}$, R. Postema ${ }^{3}$, C. Baerwald ${ }^{4}$. ${ }^{1}$ Institute for Health Economics (IfG, IfGPh); ${ }^{2}$ Bristol-Myers Squibb, Muenchen, Germany; ${ }^{3}$ Bristol-Myers Squibb, London, United Kingdom; ${ }^{4}$ Rheumatology Unit, University of Leipzig, Leipzig, Germany

Background: Studies have reported that the presence of elevated anti-citrullinated protein antibodies (ACPA)/RF levels, together with joint erosions, is associated with higher disease burden in terms of disability, and mortality in rheumatoid arthritis (RA). Abatacept has been shown to be effective in this patient population with favorable comparative data against adalimumab.(1) However, few studies have investigated the cost effectiveness of abatacept in this population to similar treatments such as TNFs.

Objectives: The objective of the study was to compare the cost-effectiveness of abatacept to adalimumab as a first bDMARD in ACPA positive RA patients who failed treatment with methotrexate (MTX) in Germany.

Methods: A decision tree model was used to estimate the cost-effectiveness, from a payer's perspective, of different treatment sequences in RA over a two year time frame. The effectiveness criteria were defined as achieving the treatment target measured by the Disease Activity Score 28 (DAS28 (CRP)<2.6; "remission"). A treatment switch to a different biologic as 2nd line and 3rd line bDMARD was allowed -in case of not achieving remission with therapy- every 6 months over a two year time period. Effectiveness data was based on randomized controlled trials (RCT) identified by an updated previous systematic literature search by the Institute for Quality and Efficiency in Health Care (IQWiG). Costs of medication and other direct medical costs were taken from a recent publication (2) and included in the analysis. Cost-effectiveness of RA treatment was investigated in ACPA positive patients in this study and presented as overall costs per day in remission. To manage uncertainty in the model, a fully probabilistic approach was used with 10,000 runs.

Results: For ACPA positive patients, treatment strategies including early treatment with abatacept had lower total costs per clinical outcome compared to later use. Figure 1 summarizes the costs per day in remission for the treatment sequences investigated: treatment sequences starting with abatacept resulted in lower costs for reaching remission (mean $330 € /$ day, range $328 €-333 € /$ day) compared to sequences starting with adalimumab (mean $384 € /$ day, range $378 €-390 € /$ day). Choice of the second or third biologic in the treatment sequences appears to have little impact on the costs per outcome.

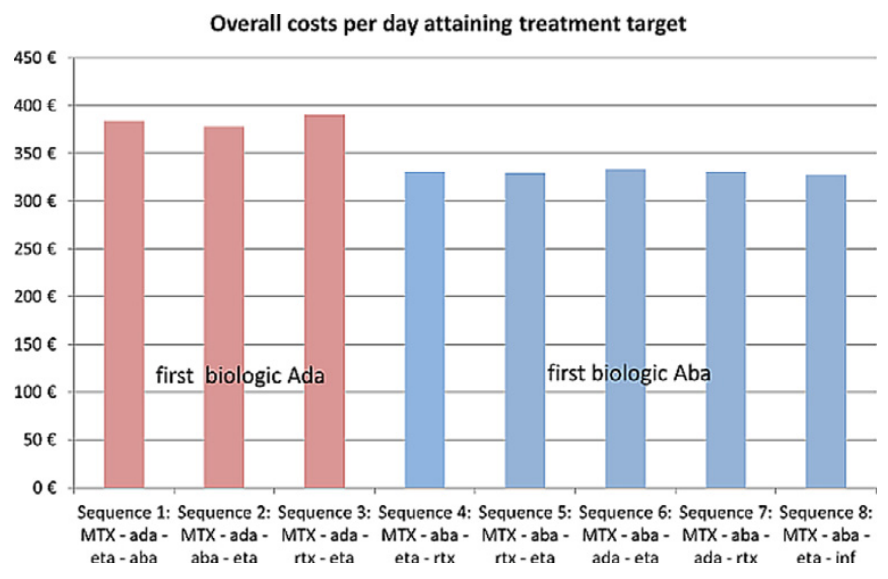

Conclusions: The results of this analysis suggest that in ACPA positive RA patients treatment with abatacept appears to be more cost-effective compared to treatment with adalimumab as a first bDMARD.

References:

[1] Sokolove J, Schiff M, Fleischmann R, et al. Annals of the rheumatic diseases. 2016 Apr;75(4):709-14. 